Du texte à la scène : langages du théâtre

\title{
Antoine Vitez : à propos de sa mise en scène d' Hamlet
}

Antoine Vitez

\section{OpenEdition}

Journals

Édition électronique

URL : http://journals.openedition.org/shakespeare/484

DOI : $10.4000 /$ shakespeare.484

ISSN : 2271-6424

Éditeur

Société Française Shakespeare

Édition imprimée

Date de publication : 1 novembre 1983

Pagination : 260-271

Référence électronique

Antoine Vitez, «Antoine Vitez : à propos de sa mise en scène d'Hamlet », Actes des congrès de la Société française Shakespeare [En ligne], 4 | 1983, mis en ligne le 01 janvier 2007, consulté le 19 avril 2019.

URL : http://journals.openedition.org/shakespeare/484 ; DOI : 10.4000/shakespeare.484 
SOCIETE FRANÇAISE SHAKESPEARE

Actes du Congrès 1982

\section{DU TEXTE A LA SCENE : Langages du Théâtre}

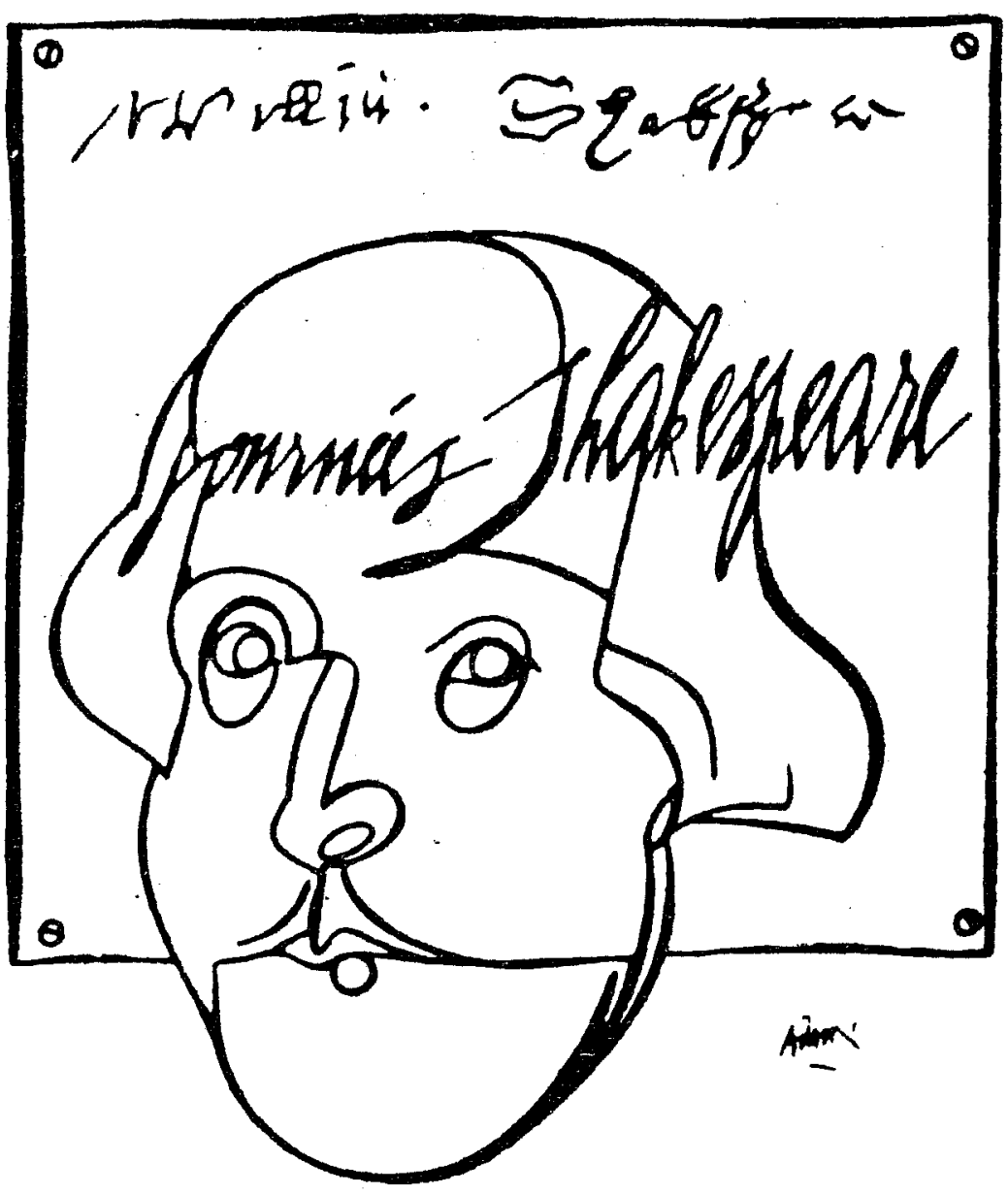

DiRecteur de la publication M.T. Jones - Davies

Publié avec le concours du Centre National de la Recherche Scientifique

JEAN TOUZOT Libraire - Editeur

38 , rue Saint-Sulpice 75278 PARIS CEDEX 061983 


\section{ANTOINE VITEZ: A PROPOS DE SA MISE EN SCENE D'HAMLET}

(Dans la retranscription ci-dessous, il a semblé opportun de suivre le déroulement de l'entretien et de privilégier, aux dépens des questions posées avec art par les congressistes, les propos, souvent intimistes, d'Antoine VITEZ, sur l'art et le métier du metteur en scène et de l'acteur)

- Pourquoi n'aviez-vous jamais monté Shakespeare ? - A.V. : ma réponse, d'ordre personnel,est liée à ma méconnaissance de la langue anglaise. J'ai horreur de l'anglais des aéroports que je parle. On peut monter des œuvres dont on ne connaît pas du tout la langue, mais la question du langage est essentielle. Ceci dit, on fait du théâtre pour des raisons de plaisir, de désir, des raisons souvent inavouées mais tout à fait avouables, non pour des raisons de devoir - du type «on ne peut pas ne pas aborder cet univers».

- Comment avez-vous choisi la traduction ? Quel texte avez-vous utilisé ? - A.V. : Le temps étant venu de former l'idée de monter Hamlet, je me suis souvenu du texte de Raymond Lepoutre, original et nouveau, joué dans un premier état de Maurice Maréchal. Nous nous sommes livrés, Raymond Lepoutre et moi, à un travail sur l'exactitude et l'élucidation du texte anglais, à une relecture, une exégèse. A partir de l'édition New Penguin et de ses commentaires scientifiques, nous avons vu les différentes solutions existantes et nous avons choisi un texte qui devait être ... un poème français, en fin de compte. Quant à la question des coupures, elle est liée à une réflexion d'homme de théâtre sur la question du temps. Dans une enveloppe de quatre heures, nous avons la possibilité d'une représentation d'environ trois heures et quart, d'où des coupes à l'intérieur de la masse du texte-en fontion de critières d'usage théâtral, dse critères forains : ce qui semble retarder l'action, ne pas apporter d'informations très excitantes pour le public. Les coupures d'usage portent 
toujours sur les mêmes points - ce qui se passe à l'intérieur de la famille Polonius, autour de Rosencrantz et Guildenstern ... parce qu'elles n'entravent pas la compréhension du texte. Hamlet est une pièce dont j'ignore le temps complet. Aussi, j'ai l'intention, le dimanche, de donner une version intégrale, quel que soit le temps du spectacle. On verrait les épisodes qu'on n'aurait pas joués pendant la semaine. En hommage à mon ami Bruno Bayen et à son Schliemann, épisodes ignorés, j'aimerais appeler ce spectacle: Hamlet, avec les épisodes ignorés.

- « Cette traduction va faire jaser», avez-vous dit ? -. - A.V. : La traduction est toujours polémique et - Henri Meschonnic l'a bien montré - profondément politique. Le livre d'Efim Etkind, sur la traduction poétique, est violent, agressif, méchant, fait pour blesser, ce qui est bien car on peut y répondre. Les partis pris par Raymond Lepoutre risquent d'apparaître comme provocants. Ils tendent à donner une idée d'un langage, d'une littérature faite de préciosité, en partie. La traduction - contre l'avis d'Efim Etkind - comme la mise en scène de théâtre, c'est donner idée de quelque chose, du temps aussi qui nous en sépare, ce n'est jamais être adéquat à quelque chose ou le représenter tel quel, c'est donner une idée de la différence (Il est faux, par exemple, que Racine soit efféminé et Corneille viril. Au contraire, le premier est direct et coupant, le deuxième plein de circonlocutions et de fioritures. L'Hippolyte, de Robert Garnier, est un exemple du goût de baroquisme dans l'écriture. Chez Garnier, l'invocation au soleil : «O grand dieu perruquier» est une invention poétique, elle fait partie d'un mode et d'une mode. C,est cela qu'a détruit Racine, par des phrases du type : «Je ne l'ai point encore embrassé aujourd'hui», qui appartient au langage commun. Le génie, c'est de faire entrer dans des alexandrins, des hexamètres, des vers de dix-huit syllabes, des phrases ordinaires. Ces phrases sont prises à l'intérieur des bornes kilométriques, de «poteaux télégraphiques» disait Claudel. Le génie c'est de trouver l'extrême simplicité, la nudité - Shakespeare est tout à l'envers, plutôt du 
côté de Garnier.)

- Pourquoi, dans Shakespeare, choisir Hamlet ? ... -A.V.: On peut feindre d'avoir une pensée consciente des actes que l'on fait, ou se livrer à l'auto-analyse. Mes raisons sont humbles. J'ai travaillé Hamlet, par petits fragments, au Conservatoire, avec mes élèves. Aussi ai-je l'impression de me promener à l'intérieur de mon souvenir quand je travaille sur cette pièce. Une raison un peu plus consciente se trouve dans les relations que j'entretenais avec mon père ... Si je m'en tiens à des raisons vraies mais conscientes, Chaillot, à cause de sa place, du passé qu'il a - avec lequel ou contre lequel je vis - me fait éprouver le besoin d'inaugurer chaque saison par une grande oeuvre philosophique - pas au sens où elle délivrerait un message - mais énigmatique, allégorique, à l'intérieur d'une recherche d'un théâtre des idées, où l'on verrait sur la scène les idées se battre entre elles (Ainsi Faust ... Je pense à une version scénique de La Célestine dont j’ai demandé la traduction à Florence Delay ).

Quant à l'art de la mise en scène - il y a plusieurs écoles; j'accumule des notes, des projets, des réflexions, des images dans des styles incompatibles. Lorsqu'à une idée s'adjoint la nécessité d'une échéance, je cherche d'abord à comprendre dans quel espace scénique je vais jouer. Ensuite, j'accumule, dans les marges du texte, des propositions de jeu - pas tellement des dessins de mise en scène, comme chez Eisenstein ou Gordon Craig, où l'on voit d'avance tout ce qui va être vu, mais l'idée des rapports des personnages entre eux, ce qui va être important dans telle séquence (pas toutes) au fur et à mesure des répétitions. La première est capitale. Dans le théâtre, il ne faut pas perdre de temps ... Je pose des structures, je me pose à moi-même des contraintes. Par exemple, si l'espace (frontal ou non) représente quelque chose, ou rien que le théâtre lui-même. C'est un choix grave. (Dans Electre, la présence de fleurs est une structure formelle). Ou encore l'âge de l'acteur qui joue Hamlet. Richard Fortuna, qui a trente ans, est le contraire d'un douteur, il ne laisse rien au hasard dans la vie ... J'ai envie, ainsi, de jouer avec des acteurs, une famille. Puis, je fais se rencontrer la pièce et la famille. 
Il y a une certaine utopie du théâtre là-dedans. Il y a des «pourquoi-pas» qui naissent tout le temps. Claudius est le résultat d'un «pourquoi-pas». On peut transporter beaucoup de choses dans le fait qu'un homme de vingt-sept ans (Aurélien Recoing, avec qui je souhaitais travailler en ce début de saison) joue Claudius, alors que la reine a cinquante ans. Leur rencontre raconte, infère une histoire. C'est le titre de la publicité de Qui Police, c'est Les Amants Maléfiques, l'histoire sordide du gigolo, frère puîné qui assassine son frère aîné et prend sa place, ceci renvoie à la dramaturgie de Shakespeare, aux histoires domestiques du kabuki, du type Arden de Faversham.

Que fait-on dans. le théâtre ? Je n'aurai, en tant qu'homme de théâtre, fait que cinq ou six choses profondes. Electre que j'ai montée, puis remontée six ans plus tard, je la recommencerai - Hamlet est un détour dans la relation que j'entretiens avec l'histoire d'Electre - toujours avec Evelyne Istria dans le rôle d'Electre. Ceci (le fait du Prince) signifie que j'ai pensé que Clytemnestre était plus jeune qu'Electre, ce que racontent deux poèmes de Yannis Ritsos - Oreste et l'Homme dans la montagne. Electre, à force d'attendre, vieillit avec la vengeance remâchée, elle maintient jusqu'à son vieil âge la certitude de la légitimité face au pouvoir usurpé, dans la Patrie et dans le lit. Elle attend toujours. Elle est celle qui sonne une cloche perpétuelle, qui empêche que l'on s'endorme. Elle est le battant. En revanche, Clytemnestre est vivante, elle aime la vie et elle aime le vivant. Elle est perpétuellement plus jeune, renouvelée. Tout ceci n'est pas nécessaire. C'est la nécessité transitoire et précaire qui est représentée au théâtre ce qui n'épuise pas le sens de l'œuvre écrite, inépuisable, donnée à tous, recevable par tous en même temps ailleurs. Mais le théâtre aura raconté cette histoire-là. Un Claudius moins illégitime, surréaliste (au sens propre), fantasmatique, qui dit "mon fils» à Hamlet alors qu'il pourrait être le fils de la même mère, voici un choc intéressant, productif de quelque chose. D'autres représentations montreront. le contraire. 
- Où vous situez-vous à l'égard des autres créations? - A.V. : Il n'y a pas de théâtre ingénu. Le théâtre, peutêtre plus que les autres arts, est polémique. Il ressemble à la politique. D'ailleurs, l'économie quotidienne du théâtre - les relations du metteur en scène avec les acteurs, la situation de l'acteur, du régisseur dans toute la ville - est au milieu d'un champ de fórces politiques, religieuses, morales, idéologiques. Il n'y a jamais rien d'innocent. L'histoire de ce qu'il faut faire - «je monte Hamlet parce que j'ai trouvé ce matin dans ma boîte à lettres le manuscrit d'un jeune homme plein de talent qui s'appelle Shakespeare et dont je n'ai jamais entendu parler» - ceci est très sot. Dans quelle mesure les hommes de théâtre sont-ils conscients de pratiquer un art qui a une histoire ? En général, on se réfère à l'histoire récente des vingt ou trente dernières années, mais le théâtre comme représentation sur la scène, l'idée que cet art a une histoire et qu'on est à l'intérieur de cette histoire, n'est pas en France ou en Angleterre, partagée par tout le monde, alors qu'elle est courante en Allemagne, en Russie, en Italie. Je suis conscient de faire des choses contre d'autres - contre ou pour. Ainsi, l'idée de pratiquer un grand théâtre des idées est dirigée contre : (1) la tendance à la démagogie populiste, qui a pour moi un visage hideux et (2) la complaisance à la disparition complète du sens et de la morale sur la scène. Entre ces deux tendances, j'ai essayé de trouver ce que pouvait être mon propre désir intellectuel. C'est à ce moment-là que je rencontre des œuvres comme $L a$ Célestine, Hamlet, Les Aïeux de Mickiewicz, que j'aimerais travailler aussi.

- Quels sont vos choix interprétatifs quant aux relations entre les personnages, la lutte de pouvoirs dans la pièce ? - A.V. : Gertrude - Madeleine Marion, pour moi l'une des plus grandes actrices du monde - me disait hier que la relation avec un Claudius jeune, d'une extrême violence charnelle, était une clé, un des noyaux d'explication de sa conduite et de toute l'histoire.Claudius devenant drogué de Gertrude, comme Edouard II l'est de son amant, 
l'on peut ainsi attribuer à Claudius des passions - sinon des sentiments. La reine est pour lui une passion. Il est intéressant que Claudius ne soit pas un simple gigolo sans scrupules mais un personnage en proie à une passion très puissante envers la reine et elle envers lui. Ceci lui donne à elle le mode d'explication de sa culpabilité et de son innocence, relativement à l'assassinat du roi - Agamemnon. Cette passion amoureuse obscurcit la conscience qu'elle a d'elle-même. Elle sait comment est mort le roi. Elle a envie de jouer cela, rétroactivement. Elle refuse d'y réfléchir parce que cet amour lui a tourné la tête, l'enivre. Nous en sommes là, pour l'instant, dans la relation entre Gertrude et moi.

Pour ce qui est du caractère énigmatique de la pièce, je préfère ne pas employer le mot de «lecture», qui présuppose que l'on a trouvé la lecture, le sens à donner à la pièce. Je m'oppose à une mise en scène qui offrirait des signes brouillés, illisibles, qui ne ferait que raconter une histoire de cape et d'épée. Nous voyons bien, d'autre part qu'il faut présenter les différents enjeux sans vouloir induire une réponse, suggérer un avenir radieux. L'avenir radieux du Danemark, si je ne me trompe, c'est l'occupation norvégienne. Toute lecture débouche sur un optimisme de la lecture. Nous sommes dans un champ de forces, Hamlet est microcosmique. Ce qu'il m'intéresse de montrer physiquement, géométriquement, sur la scène, c'est la trace au sol des trajets accomplis par les personnages, dans des rapports de domination, de subversion, de désir. C'est exactement cela le sujet pour moi. Je ne retoucherai rien à ce que m'a appris la lecture de Jan Kott. J'y ajouterai la famille - symbole ou allégorie des rapports de pouvoir, certes, - mais ce qui m'intéresse, c'est la petite histoire à l'intérieur d'une petite famille, qui est notre famille, notre histoire à vous et à moi. Bizarrement, c'est en parlant de la famille vue de près, des relations intimes du fils avec la mère, du père Polonius avec sa fille, qu'on atteindra à une audience universelle, plus large encore qu'en ne parlant que du grand mécanisme de la politique. 
- Le choix du costume va-t-il limiter le champ dans lequel vous ferez valoir l'énigme ? - A.V. : Il n'y a pas de costume zéro. Tous les costumes ont un sens au théâtre. Il y a deux grandes possibilités. On essaie d'habiller une pièce soit en costume d'Histoire, soit en costume d'Eternité. Dans le cas de l'Histoire, il y a trois possibilités : (1) le temps de la fable - Britannicus en costume romain, (2) le temps de l'écriture - Phèdre ou Britannicus en costume du XVIIe siècle, (3) le temps actuel - Le Misanthrope en habit de soirée : c'est toujours de l'Histoire, mais ce n'est pas de l'Eternité. Avec l'idée du temps actuel, on a toujours des idées actualisantes. Richard III deviendra Staline, Hitler, Pinochet, tout ce que vous voulez. Depuis quelques années, il y a une prolifération de nazis dans les pièces de Shakespeare, qui s'y prêtent. Hubert Gignoux, il y a quelques années a habillé une des deux familles de l'Horace de Corneille en paras. J'ai joué moi-même dans un Nicomède en bikinis. Le sujet, c'était les relations entre les émirs du pétrole, un mouvement de libération nationale, dans un décor de derricks. Je jouais Flaminius, c'est-à-dire l'ambassadeur des Etats-Unis. C'est bien de l'Histoire mais de l'Histoire actuelle.

Le costume d'Eternité est difficile à trouver. Il bascule irrésistiblement vers l'un des costumes historiques précédemment énumérés. Pour atteindre un effet d'éternité, il faudrait un costume en utopie et en uchronie, c'està-dire où se mélangent les styles : un empereur romain, un homme en blue-jean, un Chinois d'il y a cent ans font une utopie, et une uchronie parfaites. In très beau costume d'éternité, c'est le vêtement de travail, d'exercice, ce que Meyerhold dénomme «le vêtement de production», où l'acteur est dans une sorte de bleu. On arrive très bien à réaliser ce système dans une société totalitaire, par exemple le théâtre de camps de prisonniers ou dans les camps de concentration. Une femme qui a fait du théâtre dans un camp de concentration soviétique m'a raconté : la tenue, ce mélange de hardes et d'uniforme, que portaient les acteurs était inchangeable. Les comédiens touchaient alors à l'éternité 
$\mathrm{du}$ texte, car rien n'intervenait entre le texte, l'image que recevait le spectateur, et les actions des acteurs. Dans $L a$ Grande Illusion, une vague esquisse de décors et de costumes insuffisants crée de l'Eternité. Si nous nous mettions ainsi à jouer Hamlet, nous toucherions à l'essence. Nous serions tout nus face au texte. Un troisième système de représentation, c'est le costume imaginaire, du type Gordon Craig ou Cocteau. Créer la mode d'un royaume imaginaire, en ne l'empruntant pas à quelque chose de connu. Le paradoxe c'est qu'elle est en même temps la mode des grands aventuriers de l'époque. Il reste que le rêve, la distraction, le divertissement (au sens propre) n'est pas nécessairement donné par le costume, mais bien par les actions. Le théâtre, c'est du temps qui s'écoule et le traitement de ce temps. A quelle fréquence voit-on apparaître de belles images, entendons-nous de nouveaux mots? - c'est cela le divertissement.

- Comment concevez-vous les rapports avec le père ? Sur le plan esthétique, comment visualisez-vous le fantôme ? - A.V. : L'apparition du père est pour moi quelque chose de familier. J'ai toujours détesté la représentation du père par des truquages très savants du théâtre. La version Krejca-Svoboda utilisait un système de miroirs où le fils apparaissait dans un miroir, était son propre père. Il $\mathrm{y}$ a eu des interprétations plus ou moins sophistiquées parfois magnifiques - un rayon lumineux, une voix enregistrée, un jeu de glaces, l'hologramme (ce doit être formidable !) : des rayons fragmentés sur des surfaces réfléchissantes. On peut considérer que le père n'est pas là, qu'il n'y a rien, que le type est ventriloque, que la voix est venue d'ailleurs. Je n'aime pas ces artifices parce que j'entretiens avec mon père, que je revois la nuit, comme un mentor, des conversations construites à l'intérieur du rêve. D'où cette envie de représenter le fantôme de la façon la plus familière qui soit un diteur revêtu de l'armure qu'il portait lorsqu'il a vaincu Norvège. Un rêveur sait bien quelque part, qu'il n'est pas là. Le père devient alors le fils du fils, va peut-être essayer de le prendre dans ses bras, il ne pèsera rien du tout. 
Ceci n'est pas terrifiant du tout, c'est au contraire familier. Mon fantôme sera en chair et en os, joué par un comédien, un fantôme qui parle. La question du «vrai fantôme» évoque pour moi une anecdote. Le metteur en scène Serge Youtkevitch, à partir d'une pièce de Maïakovski - Les Bains - avait fait un film de marionnettes animées où apparaissait une poupée à la représentation du petit diable sophistiqué qu'il reproduisait dans la signature de ses lettres. Ce diable fut refusé par la censure, car ce diable n'était pas réaliste. La reconnaissance par la censure soviétique de la véritable figure du Diable, c'est la notion du «vrai fantôme». Je ne pourrai jamais condamner un metteur en scène qui représente le fantôme comme je ne sais quoi. Je n'aime pas voir un rayon de lumière passer, Hamlet se regardant dans la glace et se prenant pour son père, mais je ne trouve pas cela absurde. Il y a, c'est vrai, un gauchissement, quelque chose d'extérieur, mais qu'estce que cet extérieur à soi qui est néanmoins dans soi, qu'est-ce que c'est qu'un «vrai fantôme»? Je ne peux que répondre de manière foraine. Autrefois, le cirque Fanni, aux portes de Paris, présentait deux numéros merveilleux, l'enterrement des oies et ... Hamlet, limité à la scène entre Hamlet, Gertrude et le fantôme. Il utilisait savamment la didascalie qui nous dit que le fantôme est en chemise de nuit. La représentation devait être assez proche de la représentation originale. Ce que j'ai vu enfant, au-delà du gag culturel sur Hamlet (le fantôme portait une chemise de nuit sur son pantalon et empruntait à Hamlet une épingle à nourrice pour le remonter) c'est une scène formidable où un fils vient faire une scène terrible à sa mère et où le fantôme perd son pantalon. On voit cela dans les films de Fellini. En hommage au cirque Fanni, mon fantôme sera consistant. Je préfère un fantôme représenté par un vrai fantôme à un fantôme représenté déjà comme une lecture du fantôme. Les lectures sont possibles à l'infini - Hamlet se parlant à lui-même, le rêve, etc. A partir de l'image(rie), les théories mûrissent dans l'esprit du spectateur. Il est important d'offrir aux spectateurs les vrais 
objets théâtraux. La fêlure produite par le choix d̛un Claudius jeune est à la limite de la tension du fil interprétatif. Au-delà, l'on entre dans un autre système de théâtre - tout à fait légitime, que je me garderai de condamner, que je me garderai de dire que je ne pratiquerai pas - un système de représentation de l'art du théâtre où la poésie du spectacle, l'acte poétique des acteurs et du metteur en scène devient une œuvre dérivée, seconde, à partir d'une œuvre première, tangible. C'est cela que je ferai avec une Clytemnestre jeune, par une volonté interprétative plus violente.

- Comment s'organise votre travail avec les comédiens ? Vous arrive-t-il de vous interroger en commun sur le sens du texte ? - A.V. : Un lieu scénique, c'est d'abord un espace. Il n'y pas d'acteur de théâtre sans conscience de l'espace dans lequel il se trouve. Je ne peux pas jouer si je ne sais pas qui me regarde et d'où on me regarde. Le jeu se nourrit, littéralement, de l'espace. L'espace est consubstantiel au théâtre, ce qui fait qu'aucun archivage du théâtre n'est valable. (Il manquera toujours à l'écran la chair du théâtre, de l'acteur, c'est-à-dire l'espace). Pour imaginèr ce que je vais faire, $j$ 'ai besoin de savoir quel espace je vais utiliser. J'ai travaillé sur un espace à vue frontale - bifrontale ou tri-frontale (dans Faust, par exemple, ce qui détermine le jeu, c'est l'espace par lequel on s'échappe, 1a Nature). L'espace à l'italienne (la scène de la Comédie Française, l'Odéon) n'est qu'une des variantes de l'espace frontal. Il fonctionne à partir des lois de rapport des corps à l'intérieur d'une boîte. Yannis Kokkos a conçu une boîte à l'italienne qui serait plus ancienne que le théâtre à l'italienne, un hommage au Teatro Olimpico de Vicence, qui est entièrement fondé sur la perspective - une perspective fausse, accusée, devant des gradins à forte pente, où le corps prend des proportions étranges selon l'emplacement où il se trouve (dans le fond il est plus grand). Cette perspective, dessinée comme une épure en pointillé, c'est en même temps la représentation d'un espace entièrement fabriqué par la raison. La silhouette des acteurs, dans 
Hamlet, se détachera sur un espace entièrement blanc, sans aucun élément de représentation, d'aucun lieu, ni d'aucun temps (à la façon de la boîte blanche, dans un des escaliers du Palais - de Chaillot ! - où deux acteurs jouent en ce moment un texte de Tahar Ben Jelloun, Entretien avec M. Saïd Hammadi, ouvrier algérien, et qui est comme la préfiguration d'Hamlet). Cette boîte blanche, esthétiquement, polémique avec la Télévision- la vraie boîte à illusions, avec des vrais fantômes.

Je n'exige pas que les acteurs connaissent leur texte avant de répéter. Je ne donne que peu d'explications et ne pratique pas de travail à la table. Les vrais problèmes, théoriques, idéologiques, éthiques, qui interviennent dans le travail de n'importe quelle pièce, de la Jalousie $d u$ barbouillé à Hamlet, doivent être résolus par un choix d'actes, de détails physiques qui s'agrègent entre eux. Le travail artistique du théâtre est le contraire d'un travail discursif. Les discussions les plus profondes des acteurs entre eux, entre le metteur en scène et les acteurs portent sur des actions très simples. Chaque solution entraîne toujours une mise en scène. Les acteurs apportent des innovations, des idées, des mouvements, une manière de se déplacer, de parler. La mise en scène apporte des propositions de structure, quelques-unes impératives, comme l'espace. Le travail de choix est analogue à celui du coupeur dans la couture. Rien n'est plus beau que le geste du coupeur parce que c'est un geste définitif. Il faut avoir le courage de couper dans l'ensemble du tissu que constituent les différentes actions à choisir. Or, si une pièce d'étoffe peut se remplacer, le temps d'une journée de répétition est difficilement reproductible. De la mémoire s'est accumulée et l'on ne peut plus repartir de zéro. C'est donc le fait de jouer qui me permet de comprendre, de voir, de donner du sens. L'action théâtrale ne consiste jamais à colorier des formes en noir - à la façon des cahiers de coloriage que l'on offre aux enfants - à remplir les formes d'une pièce qui aurait du «sens». Des traces nous sont données par le temps, par l'espace au théâtre. Mais le sens ne se trouve 
Du Texte à la Scène :Langages du Théâtre.

271

pas préalablement à l'acte de théâtre.

10 octobre 1982 